\title{
Damage Detection in Grid Structures Using Limited Modal Test Data
}

\author{
Bei-dou Ding, ${ }^{1,2}$ Da-shuai Feng, ${ }^{1,2}$ Heng-lin Lv, ${ }^{1,2}$ and Xian $\mathrm{Li}^{1,2}$ \\ ${ }^{1}$ State Key Laboratory for Geomechanics and Deep Underground Engineering, China University of Mining and Technology, \\ Xuzhou 221116, China \\ ${ }^{2}$ Jiangsu Key Laboratory of Environmental Impact and Structural Safety in Engineering, School of Mechanics and Civil Engineering, \\ Xuzhou 221116, China \\ Correspondence should be addressed to Bei-dou Ding; dbdstar@163.com
}

Received 12 January 2017; Revised 13 March 2017; Accepted 2 April 2017; Published 1 June 2017

Academic Editor: Michele Betti

Copyright (C) 2017 Bei-dou Ding et al. This is an open access article distributed under the Creative Commons Attribution License, which permits unrestricted use, distribution, and reproduction in any medium, provided the original work is properly cited.

\begin{abstract}
The detection of potentially damaged elements in grid structures is a challenging topic. By using limited measured test data, damage detection for grid structures is developed by the modal strain energy (MSE) method. Two critical problems are considered in this paper in developing the MSE method to detect potential damage to the grid structure by using limited modal test data. First, an updated mode shape expansion method based on the modal assurance criterion is adopted to ensure that the modal shape obtained from the reference baseline model is reliable and has explicit physical meanings. Second, after identifying the location of the element damage by the element MSE method with expanded mode shapes, multivariable parameters denoting element damage severity are simultaneously determined. These parameters are included in the column vector and matched with the corresponding element stiffness matrix while the error tolerance value of the Frobenius norm of the column vector is undercontrolled. Finally, a threedimension numerical model of the grid structure is used to represent different damage cases and to demonstrate the effectiveness of the present method. The application of the three-dimension physical model to a full-scale grid structure is also verified. Analysis results demonstrate that the presented damage detection method effectively locates and quantifies single- and multimember damage in grid structures and can be applied in engineering practice.
\end{abstract}

\section{Introduction}

The grid structures have the advantages of light weight, high bearing capacity, high rigidity, simple and reliable connection, easy processing, and good comprehensive technical and economic indexes. They have been widely used in all kinds of public constructions such as large stadiums, gymnasiums, exhibition centers, gas stations, and industrial plants. However, grid structures in hostile environments are prone to damage because of extreme loads, fatigue, and corrosion. Grid structures continually accumulate damage throughout their service lives as a result of various environmental effects. Thus, the damage inspection and assessment of a grid structure in service are required in order to detect potential damage that may lead to a structural collapse. At present, vibration-based methods as damage detection tools instead of static measurement methods have been increasingly adopted by structural engineers because of their measurement flexibility, measurement information accessibility and easiness, and cost-effectiveness [1-3]. The basic principle of vibrationbased methods is that the changes in the physical properties of the structure will alter the dynamic characteristics of the structure, such as natural frequencies, modal damping, and mode shapes. Hence, changes in these parameters or in their combinations may be considered for localization and quantification, which are important components of damage detection [4-6].

Alvandi and Cremona [7] reviewed and summarized a few common vibration-based methods for the detection and evaluation of structural damage. Compared with previously evaluated vibration-based methods, the modal strain energy (MSE) method is more sensitive to changes in response than natural frequencies and mode shapes. MSE method is highly stable in handling noisy signals and can easily locate 
local damage. Jaishi et al. [8, 9] also reported that MSE is more sensitive to local damage than other mode shapebased indices. Stubbs et al. [10-12] first presented a damage localization method on the basis of changes in the MSE method using MSE as a damage indicator and mode shapes measured from the actual structure. Li et al. $[13,14]$ developed an improved damage localization method, specifically for the three-dimension frame structure, called the modal strain energy decomposition (MSED) method. The MSED method has two damage indicators, namely, axial and transverse damage indicators for each member of the structure. By analyzing and comparing information from both damage indicators simultaneously, the location of the damaged element can be accurately determined. Hu and Wang [15] developed a cross MSE (CMSE) method to estimate damage severity. This method is based only on the product terms obtained from two same/different modes, which are associated with mathematical and experimental models. The CMSE method does not require data on the mass distributions of the baseline and damaged structures. Implementing this method requires only the information measured by a few modes from the damaged structure. Wang et al. $[6,16,17]$ developed an iterative modal strain energy (IMSE) method to locate and quantify the damage for three-dimension frame structures especially effective when incomplete modal data are available. The merits of this new method are that both the modal frequencies and spatially incomplete mode shapes can be used. Also, the modal frequencies do not need to pair the mode shapes one by one.

MSE based methods for damage detection have been successfully applied in one-dimension or two-dimension structures. However, damage detection cases are often restricted to limited element damage stimulation and planar structure. Numerical studies and experimental verifications for a threedimensional grid structure based on synthetic data of the frequencies and mode shapes generated from the finite element models or extracted directly from the actual structure are relatively few. Grid structures are three-dimension structure and typically possess numerous elements and are geometrically symmetrical; the natural vibration frequencies and mode shapes of grid structures are highly intensive. Usually, the traditional MSE methods for the damage detection are required to achieve mass normalized mode shapes or spatially complete mode shapes. Considering that the grid structure possesses many degrees of freedom (DoFs) and only a limited number of sensors can be placed in it, hence, the modal expansion technique $[15,16]$ is used to get the spatially complete mode shapes of grid structure from limited measuring sensors applied to the grid.

The main objective of the present paper is to develop MSE method for damage localization and severity estimation of the grid structures of numerical and actual models by using limited modal test data. First, a physical model of a threedimension grid structure is built, modal testing is conducted, and the damage localization detection indicator and damage severity detection based on MSE methods are validated with numerical data. Second, the incomplete spatial mode shapes measured from the dynamic test of grid structure due to quantitative limitation of sensors are then expanded to the complete mode shapes using the modal expansion technique. Third, a full-scale grid structure with different corrosion rates of the elements is used to demonstrate the effectiveness of the damage localization and severity detection of threedimensional structure. This paper is arranged as follows. First, a mode shape expansion method based on the modal assurance criterion (MAC) is adopted to ensure that the measured incomplete mode shapes from the limited modal test data in the lab test are expanded to the complete mode shapes, so that the modal shapes obtained from the analytical model are reliable and have explicit physical meanings. Second, damage localization and severity estimation of the grid structures based on MSE method are developed with analytical modal frequencies and expanded mode shapes. At the same time, both single and multiple damage cases for numerical models of the grid structures are set up with different levels of severity to verify the effectiveness of damage localization. Finally, to demonstrate the capabilities of the developed damage severity estimation algorithm, a grid structure physical model is constructed and tested with different corrosion rates of the members. Modal parameters are identified and used for the damage detection methods. Results indicate that the MSE method is effective on damage localization and severity detection of three-dimensional grid structure and can be applied in engineering practice.

\section{Methodology}

2.1. Mode Shape Expansion. The measured mode shapes of the grid structure due to quantitative limitation of sensors are incomplete and they should be expanded to finite element model DoFs. Before the mode shape expansion, the comparison between the analytical modal shapes from the finite element model analysis and the measured mode shapes from the experimental modal analysis is performed by calculating the MAC value. Equation (1) is the formula for calculating the MAC value of the $i$ th modal shape.

$$
\operatorname{MAC}_{i}=\frac{\left(\Phi_{a i}^{T} \cdot \Phi_{d i}\right)^{2}}{\left(\Phi_{a i}^{T} \cdot \Phi_{a i}\right)\left(\Phi_{d i}^{T} \cdot \Phi_{d i}\right)},
$$

where $\Phi_{a i}$ denotes the $i$ th analytical modal shape and subscript " $d$ " indicates modal shape associated with the damaged structure. High MAC values indicate greater correlation between the analytical modal shapes and the measured incomplete mode shapes. If the MAC value is greater than or equal to 0.97 , the analytical modal shapes can then be used directly without any further improvement. Then, the measured incomplete mode shapes could be expanded to complete the mode shapes by using the mode shape expansion method suggested by Ren and Chen [9]. In this method, the measured modes are assumed to be a linear combination of the analytical modes, and the unmeasured modes can be expanded by using a transformation coefficient, which is given by the following relationship:

$$
\left(\begin{array}{c}
\Phi_{d 1(n \times p)} \\
\Phi_{d 2((N-n) \times p)}
\end{array}\right)=\left(\begin{array}{c}
\Phi_{a 1(n \times l)} \\
\Phi_{a 2((N-n) \times l)}
\end{array}\right) \alpha_{l \times p},
$$


where $N$ is the number of DoFs, $n$ is the number of measured DoFs, $p$ is the number of identified mode shapes, $l$ is the number of mode shapes used in the expansion, and $\alpha$ is the transformation coefficient. As long as $n \geq l$, coefficient $\alpha$ can be obtained by using a least-squares technique

$$
\alpha=\left(\begin{array}{ll}
\Phi_{a 1}^{T} & \Phi_{a 1}
\end{array}\right)^{-1}\left(\begin{array}{ll}
\Phi_{a 1}^{T} & \Phi_{d 1}
\end{array}\right)
$$

The mode shapes of the unmeasured DoFs, that is, $\Phi_{d 2}$, can then be easily computed by using (2). After the measured mode shapes are expanded according to the analytical mode shapes of the undamaged structure, the updated mode shapes can be used in the process of damage localization and severity detection based on MSE method with explicit physical meaning and small iteration error.

2.2. Damage Localization Indicator. The indicator initially comes from the Stubbs index, which is based on traditional MSE method and developed by Stubbs et al. (1995). Yang (2003), Li et al., and (2006) Wang et al. (2014) further developed two damage indicators, the axial damage indicator and the transverse damage indicator based on the modal strain energy decomposition (MSED) method for threedimensional frame structures. In this section, the damage localization indicator based on MSE method is introduced for the damage localization of the grid structure.

Consider that the grid structure mainly bears the axial force, and the $i$ th modal strain energy of the $j$ structural member can be expressed as

$$
U_{i j}=\frac{1}{2} \phi_{a i}^{T} K_{j} \phi_{a i}
$$

where $K_{j}$ is the axial stiffness matrix terms of member $j$. The whole modal strain energy of the grid structure should be the sum of all the members, so for a linear, the $i$ th modal strain energy of the undamaged grid structure based on the finite element model is given by

$$
U_{i}=\frac{1}{2} \phi_{a i}^{T} K \phi_{a i},
$$

where $K$ is axial stiffness matrix of the grid structure. The fraction of the $i$ th modal energy that is concentrated in the $j$ th member (i.e., the sensitivity of the $j$ th member to the $i$ th mode) is defined as

$$
F_{i j}=\frac{U_{i j}}{U_{i}} .
$$

If the subscript " $d$ " is used to represent the modal parameters in (4) to (6) for a damaged structure, the damaged sensitivity of the $j$ th member to the $i$ th mode can be written as

$$
F_{i j}^{d}=\frac{U_{i j}^{d}}{U_{i}^{d}}
$$

in which the quantities $U_{i j}^{d}$ and $U_{i}^{d}$ are given by

$$
U_{i j}^{d}=\frac{1}{2} \phi_{d i}^{T} K_{j} \phi_{d i}
$$

$$
U_{i}^{d}=\frac{1}{2} \phi_{d i}^{T} K \phi_{d i}
$$

Assume that the damage only changes the stiffness of the member, but not the mass of the structure, and the modal strain energy for each $i$ th mode before and after damage remains constant: that is, $U_{i}=U_{i}^{d}$. Meanwhile, assume that the modal sensitivity for the $i$ th mode and the $j$ th location is the same for both undamaged and damaged structures: that is, $F_{i j}=F_{i j}^{d}$.

The fraction indicator of the $i$ th modal energy could be divided by zero possibly; to overcome the situation, according to the above assumption, the axis of the modal sensitivity should be shifted by one: that is, $F_{i j} \rightarrow F_{i j}+1, F_{i j}^{d} \rightarrow F_{i j}^{d}+1$; then

$$
\frac{F_{i j}^{d}+1}{F_{i j}+1}=\frac{U_{i j}^{d} / U_{i}^{d}+1}{U_{i j} / U_{i}+1}=\frac{\left(U_{i j}^{d}+U_{i}^{d}\right) U_{i}}{\left(U_{i j}+U_{i}\right) U_{i}^{d}}
$$

Substituting (4), (5), and (8) into (9), a damage indicator $Z_{i j}$ for $i$ th mode of the $j$ th member is obtained by

$$
Z_{i j}=\frac{F_{i j}^{d}+1}{F_{i j}+1}=\frac{\left(\phi_{d i}^{T} K_{j} \phi_{d i}+\phi_{d i}^{T} K \phi_{d i}\right)}{\left(\phi_{i}^{T} K_{j} \phi_{i}+\phi_{i}^{T} K \phi_{i}\right)} \cdot \frac{\phi_{i}^{T} K \phi_{i}}{\phi_{d i}^{T} K \phi_{d i}} .
$$

If the mode of the actual test structure has $N$ order measured modes obtained in the dynamic test of the grid structure, a damage indicator $Z_{i j}$ for the $j$ th member is defined by

$$
Z_{j}=\frac{\sum_{i=1}^{N}\left(\phi_{d i}^{T} K_{j} \phi_{d i}+\phi_{d i}^{T} K \phi_{d i}\right) \cdot \phi_{i}^{T} K \phi_{i}}{\sum_{i=1}^{N}\left(\phi_{i}^{T} K_{j} \phi_{i}+\phi_{i}^{T} K \phi_{i}\right) \cdot \phi_{d i}^{T} K \phi_{d i}}
$$

Then, after normalization of the damage indicator $Z_{j}$, the damage location index can be used to detect the damage localization of the grid structure members.

2.3. Damage Severity Detection. After the damaged elements are identified with damage localization indicator, a modified MSE method is developed to determine damage severity of the elements in the grid structure. The modal eigenvalue analysis of the baseline model and damaged model of the grid structure can be written as follows:

$$
\begin{gathered}
K \Phi_{i}=\lambda_{i} M \Phi_{i}, \\
K_{d} \Phi_{d i}=\lambda_{d i} M_{d} \Phi_{d i},
\end{gathered}
$$

where $M$ and $K$ are the mass and stiffness matrices of the undamaged structure, respectively, $\lambda_{a i}$ denotes the $i$ th analytical modal eigenvalue, and subscript " $d$ " indicates the values associated with the damaged structure.

Structural stiffness is generally decreased because of section reduction of local elements under corrosion environment. Assume that the mass of elements is unchanged: that is, $M=M_{d}$, and the structural element damaged 
stiffness for the $i$ th element can be expressed as a decrease of the undamaged stiffness. Therefore, in the local coordinate system, the local stiffness matrices of the damaged elements are assumed as linear combination between undamaged and damaged stiffness matrices; that is,

$$
k_{d i}=k_{i}+\beta_{i} k_{i} \quad\left(-1<\beta_{i}<0\right),
$$

where $k_{d i}$ and $k_{i}$ are the $i$ th stiffness matrices of the damaged and undamaged local element stiffness matrix, respectively, and $\beta_{i}$ represents the damage extent of the $i$ th damaged element. The global stiffness matrix of the damaged structures can then be written as follows:

$$
K_{d}=K+\sum_{i=1}^{N_{d}} \Delta K_{i}=K+\sum_{n=1}^{N_{d}} \beta_{n} K_{l_{n}} \quad\left(-1<\beta_{n}<0\right),
$$

where $N_{d}$ is the total number of damaged members, $\beta_{n}$ and $l_{n}$ represent the damage extent and the number of damaged elements, respectively, and $K_{l_{n}}$ is the stiffness matrix of the $l_{n}$ th element. By using (15), damage severity can be estimated by an available damage detection method.

By premultiplying (12) by $\Phi_{d i}^{T}$ and (13) by $\Phi_{i}^{T}$ and considering the symmetry of the mass and stiffness matrices, the following can be determined:

$$
\Phi_{i}^{T} K_{d} \Phi_{d i}=\frac{\lambda_{d i}}{\lambda_{i}} \Phi_{i}^{T} K \Phi_{d i}
$$

Substituting (15) into (16) yields the following:

$$
\sum_{n=1}^{N_{d}} \beta_{n} \cdot C_{n, i}=b_{i}
$$

where

$$
\begin{aligned}
\beta & =\left\{\begin{array}{lll}
\beta_{1} & \beta_{2} \cdots & \beta_{N_{d}}
\end{array}\right\}, \\
C_{n, i} & =\Phi_{i}^{T} K_{l_{n}} \Phi_{d i}, \\
C_{i} & =\Phi_{i}^{T} K \Phi_{d i}, \\
b_{i} & =\left(\frac{\lambda_{i}}{\lambda_{d i}}-1\right) C_{i},
\end{aligned}
$$

$\beta_{n}, C_{n, i}$, and $C_{i}$ represent, respectively, the corresponding element damage severity, the MSE column vector in stiffness matrix $K_{l_{n}}$, and the total structural MSE for structural stiffness $K$ between the undamaged and damaged structures for the $i$ th mode.

Equation (17) can then be simplified as follows:

$$
C \beta=b .
$$

$m$ equations can be derived from (17) when $m$ modes are available for the undamaged and damaged structures. In (19), $C$ is a $m \times N_{d}$ matrix, and $\beta$ and $b$ are column vectors of size $N_{d}$ and $m$, respectively. When $m$ is greater than or equal to $N_{d}$, a least-squares approach can be conducted to solve $\beta$. The estimate of $\beta$, denoted as $\widehat{\beta}$, is written as follows:

$$
\widehat{\beta}=\left(C^{T} C\right)^{-1} C^{T} b \text {. }
$$

To solve (20), the mode shapes at the full coordinates of the damaged structures are required. These mode shapes can be calculated by the mode shape expansion technology with high MAC values, described in Section 2.2. To estimate the damage extent of a structural element, an iterative approach is adopted. In this approach, the mode shapes of the damaged structure are associated with damage severity by the following:

$$
\Phi_{d i}=\Phi_{d i}\left(K_{d}, M\right)=\Phi_{d i}\left(K+\sum_{n=1}^{N_{d}} \beta_{n} K_{l_{n}}, M\right) .
$$

The errors of the damage severity column vector between two iterations are space vectors and expressed in the form of a Frobenius norm function, shown in (22), and are used as the judgment condition in the process of the iteration.

$$
\left\|\beta^{(k)}-\beta^{(k-1)}\right\|_{F}^{2}
$$

2.4. Implementation Process of Damage Detection. Then, the damage localization and damage severity detection can be estimated iteratively as follows based on the methods in Sections 2.3 and 2.4 .

Step 1. A finite element mode shape $\Phi_{i}$ and modal eigenvalue $\lambda_{i}$ of the undamaged structure are obtained by finite modal analysis. The measured incomplete mode shapes $\Phi_{d i}$ are expanded into complete mode shapes $\Phi_{d i}^{0}$ according to the finite element mode shape $\Phi_{i}$ by using $(2) . \Phi_{d i}^{0}$ can then be used in the subsequent process of damage localization and severity detection.

Step 2. A damage localization indicator is calculated by using (11). After normalization of the damage localization indicator, the normalized indicator is used to identify the damaged elements in the grid structure.

Step 3. After the damage elements are identified according to the damage localization indicator, the vector of the estimated damage severity $\beta^{(1)}$ is calculated by using (20) with the expanded $\Phi_{d i}^{(0)}$. Subsequently, the damaged mode shape $\Phi_{d i}^{(k-1)}$ for $k=2,3, \ldots$ is computed by using (21) with the vector of estimated damage severity $\beta^{(k-1)}$. Damage severity $\beta^{(k)}$ is estimated by using (20) on the basis of $\Phi_{i}^{(k-1)}$ (given $k=2,3, \ldots)$ sequentially.

Step 4. The iteration termination condition is set. Step 4 is repeated until the Frobenius norm $\left\|\beta^{(k)}-\beta^{(k-1)}\right\|_{F}^{2} \leq$ tol, where tol is a predetermined threshold.

\section{Numerical Studies on Damage Detection in Grid Structures}

3.1. The Finite Element Model of Grid Structures. To verify the foregoing damage method, a $2 \times 2$ grid structure that consists of 32 steel tubular members with a length of $3.0 \mathrm{~m}$, a width of $3.0 \mathrm{~m}$, and a height of $1.0 \mathrm{~m}$ was numerically 


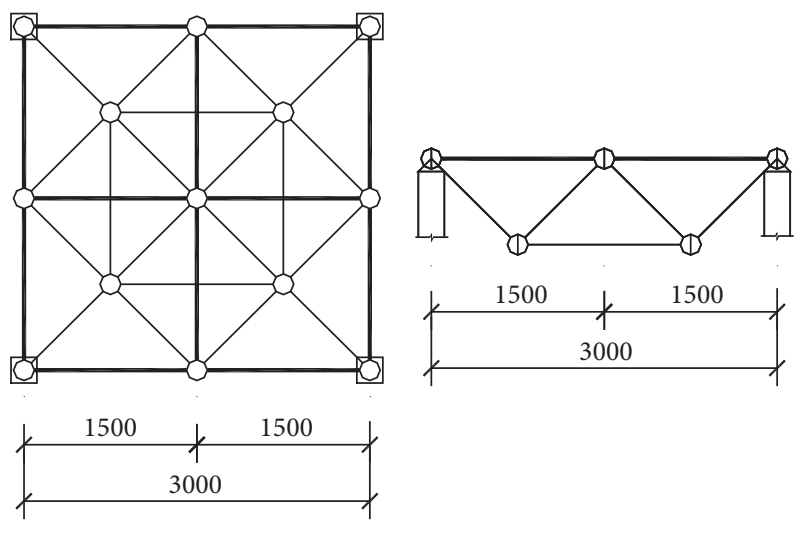

Figure 1: Experimental model of a baseline grid structure.

analyzed. This structure is shown in Figure 1. The members are assumed to be axial tension-compression members that have been pin-ended and pin-braced against the supports in the ground, and all the members have similar tube crosssections $\phi 42 \mathrm{~mm} \times 3.25 \mathrm{~mm}$. The material properties of the steel tubular members include elastic modulus $E=210 \mathrm{GPa}$, Poisson's ratio $\nu=0.3$, mass density $\rho=7850 \mathrm{~kg} / \mathrm{m}^{3}$, and yield strength $=235 \mathrm{MPa}$. Loads are applied to the nodes of the top chord. The design parameters of the test grid structure are listed in Table 1.

3.2. Simulated Damage Cases of Grid Structure. To realize the aforementioned research concepts, six damage cases are numerically analyzed, which are listed in Table 2. Damage Cases $\mathrm{A}$ to $\mathrm{C}$ are used to simulate the single-member damage, with a $20 \%$ stiffness reduction. Damage Cases D to F simulate the multimember damage, with a $20 \%$ stiffness reduction. Despite slight damage, the structure remained linear, and the damage was often exhibited by deterioration in the stiffness of the members rather than by its integral deterioration.

3.3. Damage Detection Analysis. The damage indicators can be calculated by using (11). The element damage indicators of single- and multimember damage detection are listed in Tables 3 and 4, respectively. Node and element numbering are indicated in the corresponding damaged model of the grid structure.

Table 3 shows the damage indicator for damage Case A wherein the first six modal frequencies and mode shapes are used. The localization of damaged member 23 is not uniquely estimated, given the geometric system of the grid structure in the simulation. The damage indicators of members 23 and 24 are close to -0.0076 , and both their damage severities are estimated to be $19.9 \%$. These members are adjacent to each other and are situated in symmetrical areas of the grid structure. Therefore, members 23 and 24 are concurrently identified as damaged and must be validated further. The damage indicators of members 1 and 32, which are diagonal and bottom chord members in damage Cases B and C, are close to -0.018 and -0.0077 , respectively, and the estimated damage severities are $19.81 \%$ and $20.11 \%$. Damage Cases B and
$\mathrm{C}$ are also shown in Table 3 and can be exclusively identified, although members 1 and 32 are located in symmetrical areas of the grid structure. The support constraint conditions presumably alter the symmetrical physical conditions and the mode shapes.

Table 4 presents the damage indicators for damage Cases $\mathrm{D}, \mathrm{E}$, and F, wherein multielement damage scenarios are simulated. In damage Case $\mathrm{D}$, the locations of damaged members 23 and 26 are not uniquely estimated because of the geometric system of the grid structure in the simulation. The damage indicators of members 24 and 25 are close to -0.0076 and their estimated damage severities are $19.92 \%$ and $20.05 \%$, respectively. These two members are simultaneously identified as damaged. Members 23 and 24 are adjacent to each other, whereas members 25 and 26 are situated in symmetrical areas of the grid structure. Therefore, these two members are also concurrently identified as damaged and must be validated further. The damage indicators of members 1 and 9 and 31 and 32, which are diagonal and bottom chord members, respectively, are close to -0.06 and -0.014 . The estimated damage severities are $19.85 \%$ and $19.78 \%$.

It should be pointed out that, in the damage Case A, chord element 23 and chord element 24 have the same node 2 and the constrained joints of node 1 and node 3 are symmetrical, so element 23 and element 24 have the same mode shape. Similarly, in the damage Case D, element 23 and element 24 and element 25 and element 26 have the same nodes 2 and 4, respectively, and the constrained joints of node 1 , node 3 , and node 7 are also symmetrical, so element 25 and element 26 also have the same mode shape. There is existing symmetry phenomenon in damage identification based on MSE method. Then, element 1 and element 32 can be accurately identified because the element constraints and geometric conditions are different.

In fact, there are a large number of elements and nodes in the actual grid structure. If the damage region of the grid structure is located, it is enough to identify the damage in the grid structure. Take into account that the axial force is mainly transmitted at nodes and elements in the grid structure. In the further work, the more measured locations are supposed to be added in the middle part of the damage localized member; the local vibration modes of the member can be obtained; then, the damage localization of the member could be identified exactly according to the local information.

\section{Experimental Validation}

To verify the proposed damage detection method, a fullscale model of the grid structure is fabricated. This model has identical geometrical properties with those described in the numerical study. The steel grid structure model is undergoing one-year corrosion treatment in advance and displays a certain thickness tolerance measured by using a pachymeter. This measurement is compared with the results acquired by damage detection methods.

According to the measurement results, the average corrosion deviation rate is approximately $10 \%$, except that the corrosion deviation rates of member 14 and member 15 are $23 \%$ and $32 \%$, respectively. Considering the average $10 \%$ corrosion 
TABLE 1: Design parameters of a test grid structure.

\begin{tabular}{lccc}
\hline Plane size & $3 \mathrm{~m} \times 3 \mathrm{~m}$ & Number of bays & $2 \times 2$ \\
Height & $1 \mathrm{~m}$ & Support type & Pinned \\
Member dimension & $42 \mathrm{~mm} \times 3.25 \mathrm{~mm}$ & Bolt ball diameter & 80 \\
\hline
\end{tabular}

TABLE 2: Simulated cases of grid structure damage.

\begin{tabular}{lccc}
\hline Damage type & Damaged member & Element number & Damage extent \\
\hline A & Top chord & 23 & $20 \%$ \\
B & Diagonal member & 1 & $20 \%$ \\
C & Bottom chord & 32 & $20 \%$ \\
D & Top chord & 23,26 & $20 \%$ \\
E & Diagonal member & 1,9 & $20 \%$ \\
F & Bottom chord & 31,32 & $20 \%$ \\
\hline
\end{tabular}

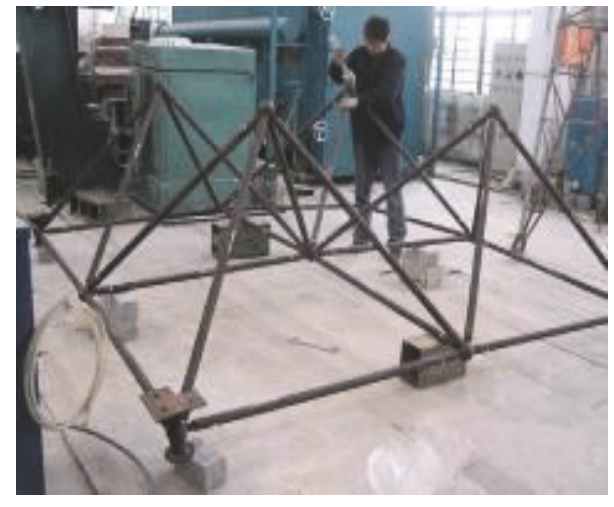

FIGURE 2: Fabrication of diagonal members.

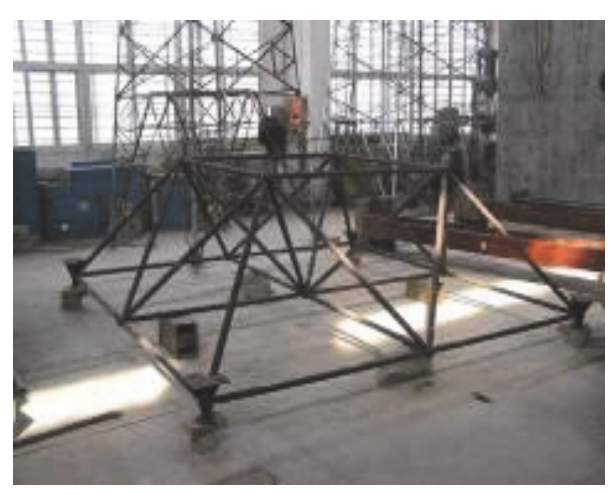

Figure 3: Assembled grid structure.

deviation rate, a $10 \%$ stiffness loss is supposed. Then the grid structure model with $10 \%$ stiffness reduction is treated as the baseline model and the dynamic modal parameters are analyzed by the finite element method. The relative damage rates of members 14 and 15 are the identification targets of damage severity.

The process of grid structure fabrication and the assembled grid structure are shown in Figures 2 and 3, respectively. The assembled grid structure is installed on the test platform, and the load is applied. The schematic of the specimen

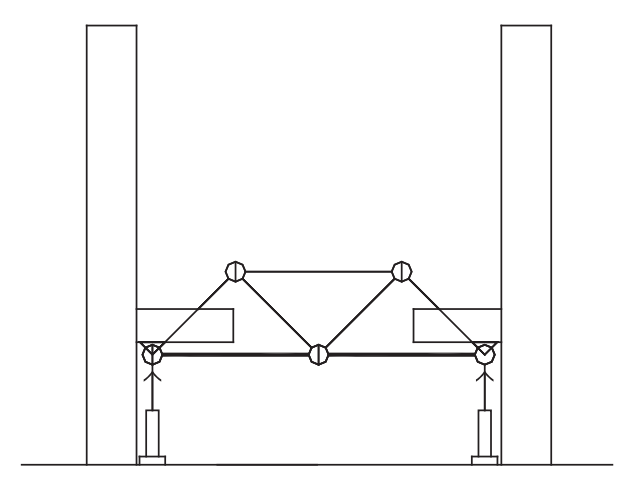

FIgURE 4: Schematic of the specimen loading device.

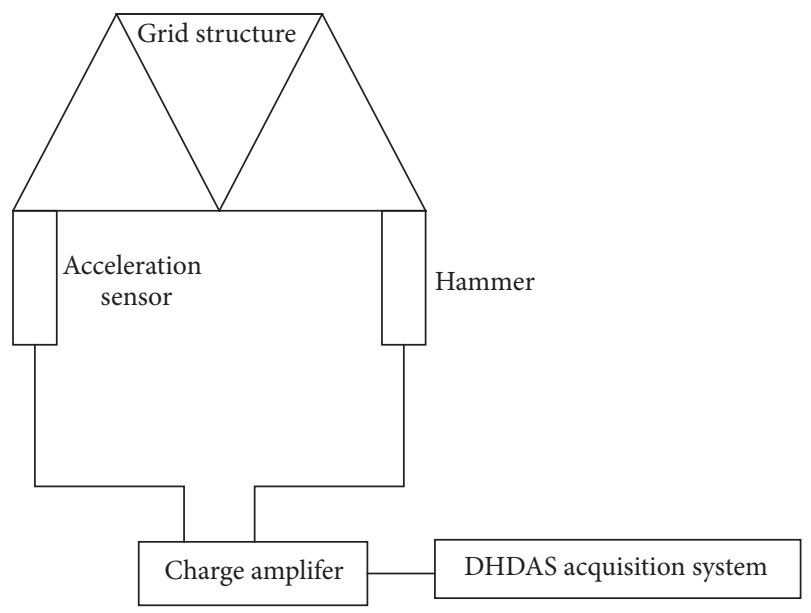

FIGURE 5: DH5935 acquisition system diagram.

loading device is illustrated in Figure 4. The diagram of the acquisition system under hammer excitation is exhibited in Figure 5. The flat-hinged support joint of the grid structure that uses a bearing joint is presented in Figure 6 . The grid nodes are bolt ball joints, and bolt joints with 4, 6, 7, and 9 holes are applied according to the stress characteristics of the grid structure (Figure 7). 
TABLE 3: Damage localization detection in a single element.
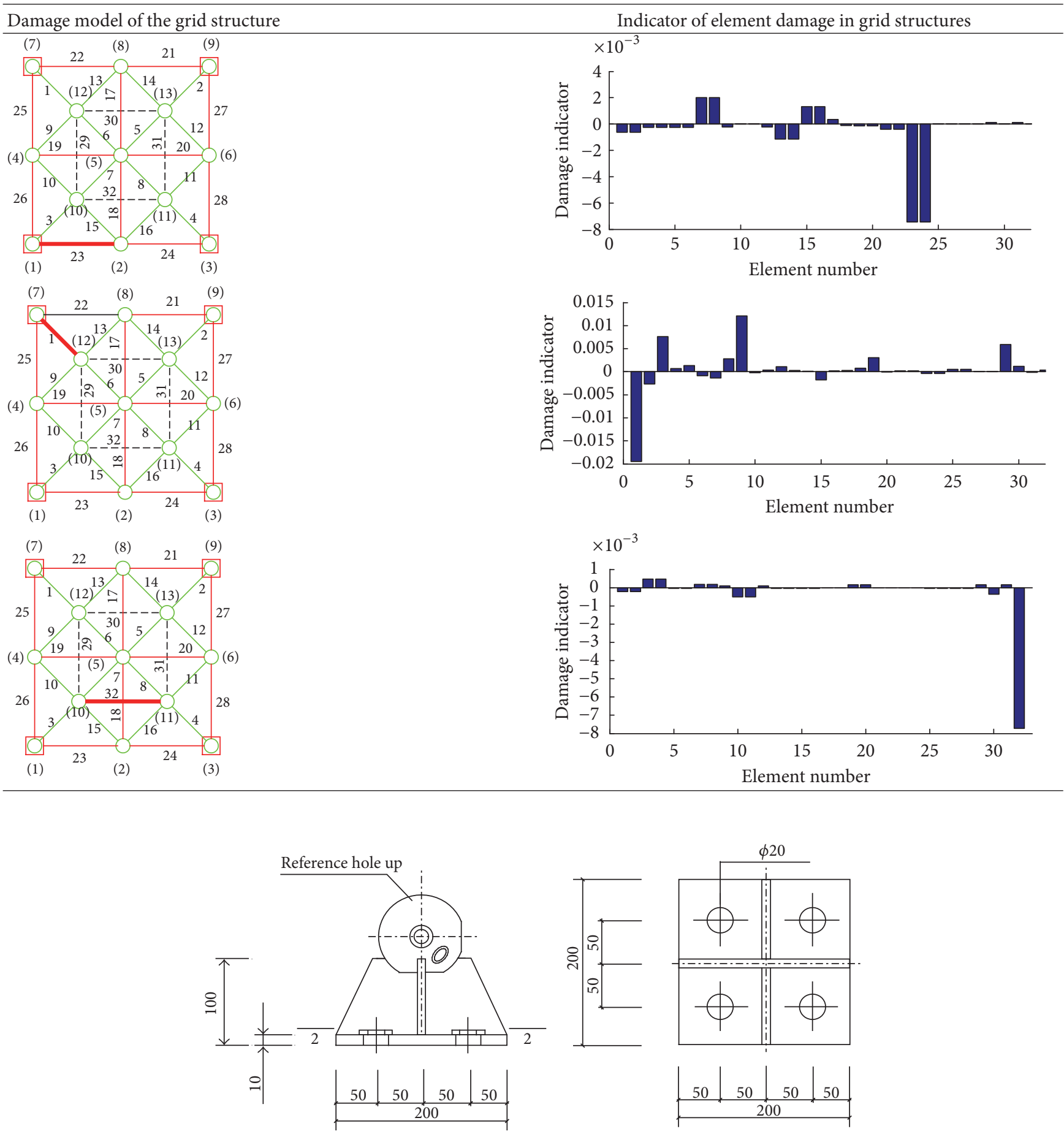

Figure 6: Details of the bearing joint.

The theoretical analysis and experimental results reveal that the natural vibration frequencies of the grid structures are intensive and difficult to identify. By using finite element analysis, the characteristics of different mode shapes in the grid structure are simulated. The first mode shape is a horizontal torsion shape, the second mode shape is an overall upward-sloping shape, and the last three mode shapes are antisymmetric and reverse shapes. While the vibration characteristics of the grid structure can be identified by finite element analysis, the actual modal parameters of the grid structure can be identified by a modal test. A comparison of the frequency results determined by finite element modal analysis and those by experimental modal analysis is presented in Table 5. Both frequency values of the grid structures 

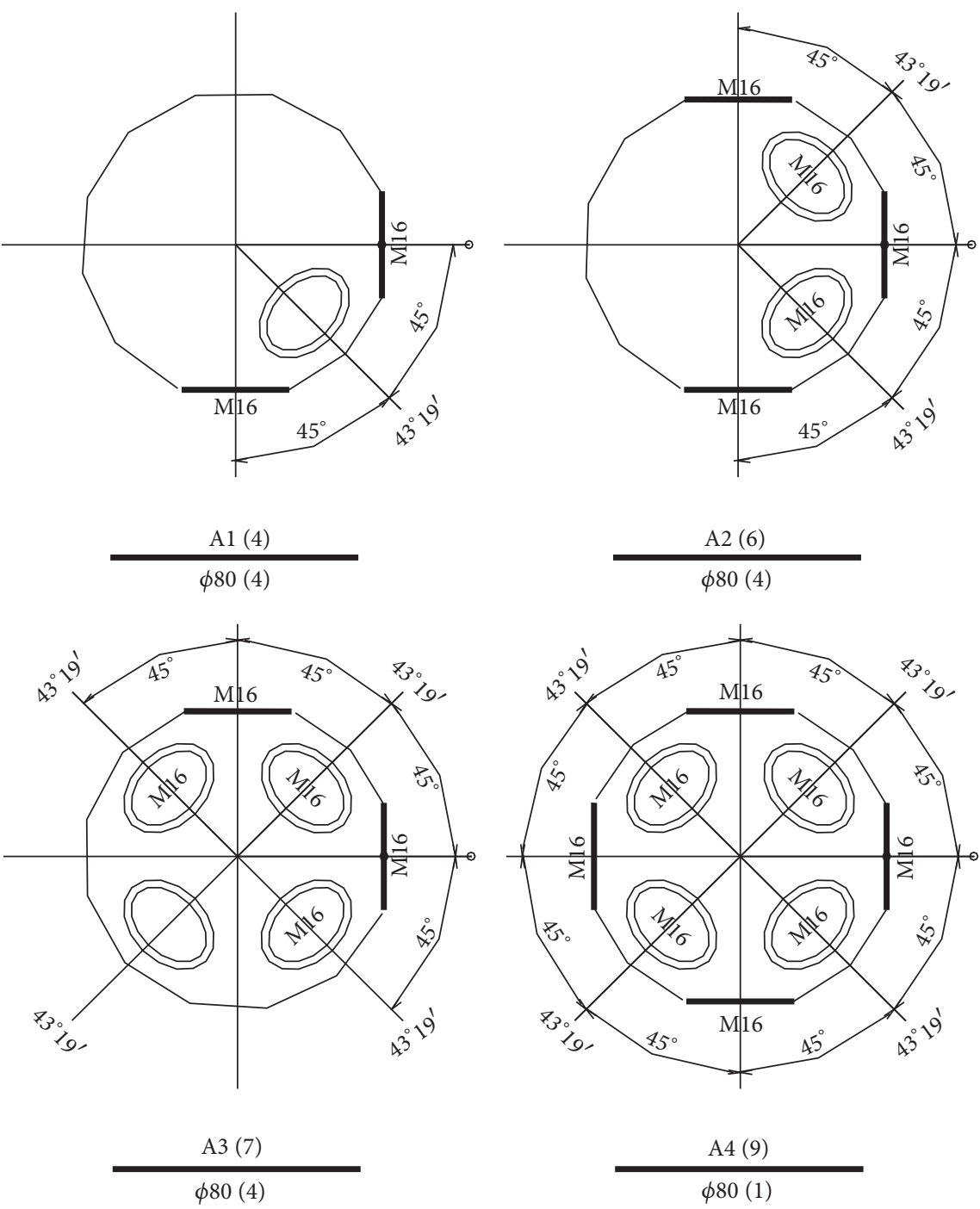

Figure 7: Detailed process of constructing the bolt joints of the grid structure.

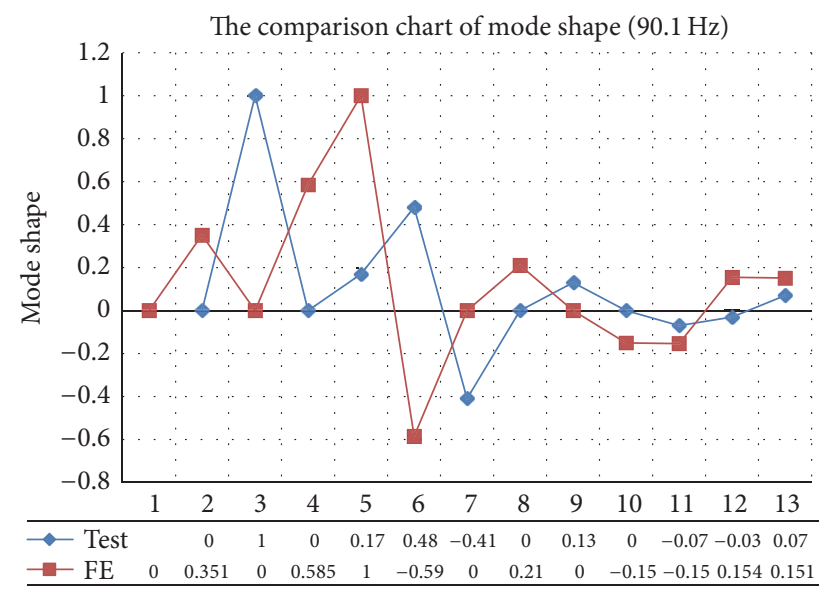

(a) $f=90.1 \mathrm{~Hz}$

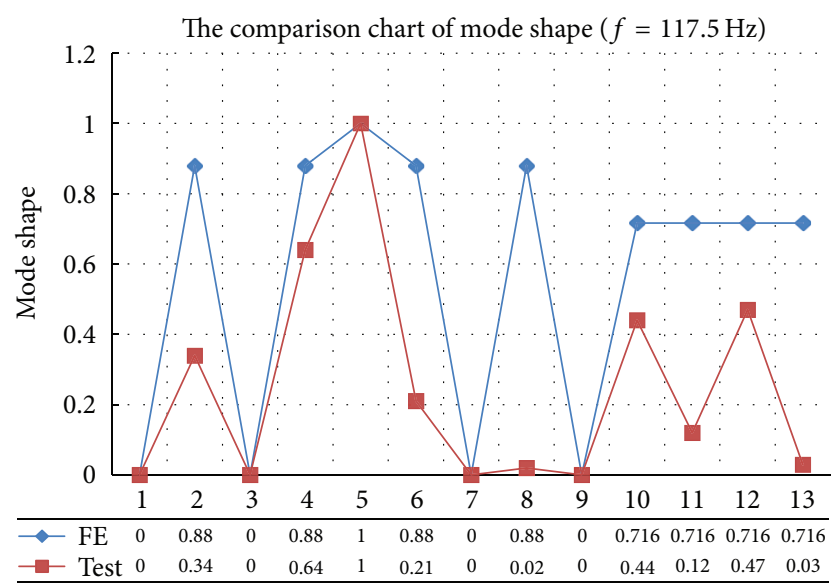

(b) $117.5 \mathrm{~Hz}$

FIGURE 8: Comparison chart of mode shape. 
TABLE 4: Damage localization detection in multiple elements.

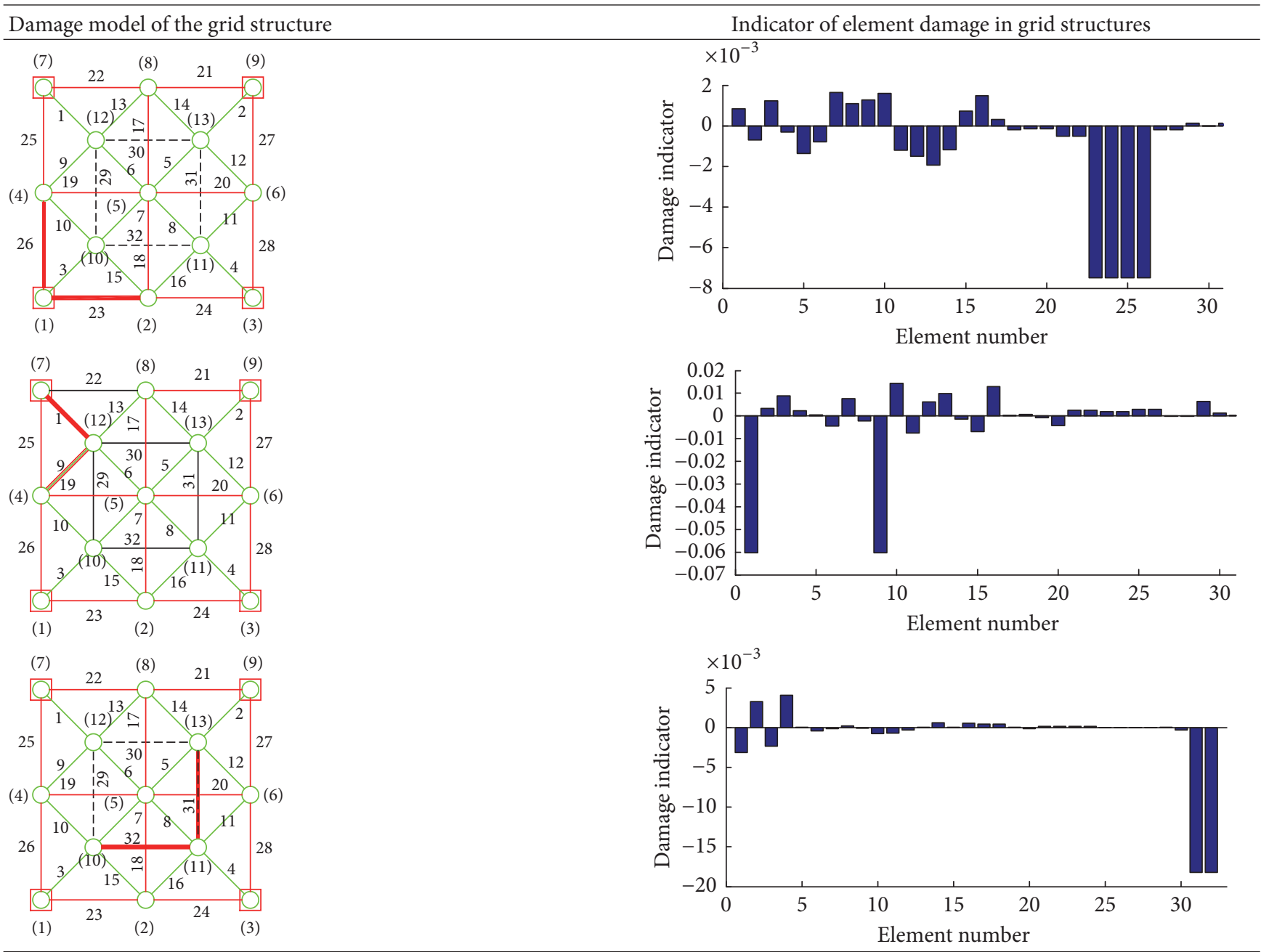

TABLE 5: Grid structure frequencies $(\mathrm{Hz})$.

\begin{tabular}{lcccc}
\hline Mode & Finite element analysis $(\mathrm{Hz})$ & Test result $(\mathrm{Hz})$ & Differences in frequencies $(\%)$ & Characteristics \\
\hline 1 & 90.1 & 89.84 & 0.289 & Horizontal torsion \\
2 & 117.5 & 117.19 & 0.264 & Overall upward-sloping \\
3 & 246.09 & 248.29 & -0.894 & Antisymmetric reverse \\
4 & 307.7 & 304.69 & 0.978 & Antisymmetric reverse \\
5 & 377.5 & 380.86 & -0.890 & Antisymmetric reverse \\
\hline
\end{tabular}

are very close, and the differences in frequencies from modes 1 to 5 are $0.289 \%, 0.264 \%,-0.894 \%, 0.978 \%$, and $-0.890 \%$, respectively.

The first two mode shapes are compared in Figures 8(a) and $8(\mathrm{~b})$. The mode shapes of the grid structure as determined by finite element analysis and experimental modal analysis are similarly plotted. These shapes are similar as a whole, although local changes are induced by the loss of element sections in the grid structures. Only two modes are compared and their frequencies from finite element analysis are 90.1 and $117.5 \mathrm{~Hz}$ while their MAC values are the same at 0.97 . These findings show that the mode shapes between the experiment and finite element modals have a high relationship.

To evaluate the effectiveness of the presented damage method by using measured data obtained from lab dynamic test, damage quantification is iteratively analyzed. After determining damaged members 14 and 15 by using (11), damage severity $\widehat{\beta}$ is estimated by using (20). Damage identification is applied to all grid structure members; a series of iterations are then conducted. Finally, the relative damage severities of members 14 and 15 are calculated as $13.1 \%$ and $22.5 \%$, 

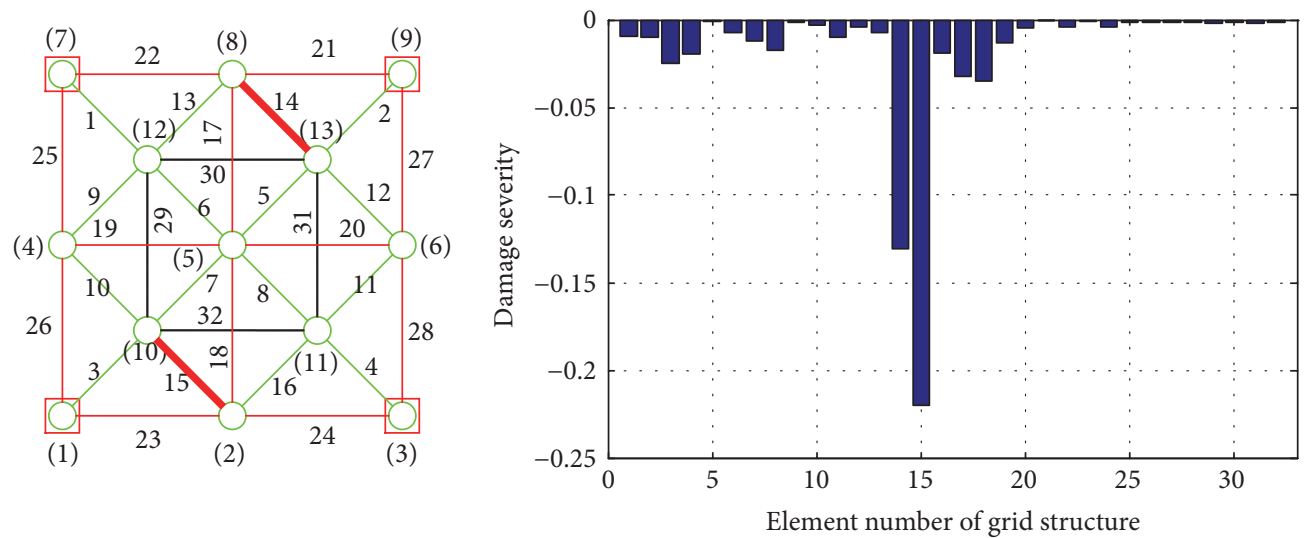

FIGURE 9: Damage detection result for a full-scale grid structure.

respectively (Figure 9). The relative error in the estimation of damage severity increases with the identified numbers in the grid structure.

\section{Conclusions}

This study develops modal strain energy (MSE) method for damage localization and severity estimation of the grid structures of numerical and actual models by using limited modal test data. The following conclusions may be drawn from this study:

(1) By comparing the MAC values between analytical modal shapes from the finite element analysis and the measured mode shapes from the experimental modal analysis of the actual grid structure, the matched analytical mode shapes of the baseline model can be acquired, only if the MAC value is greater than 0.97 . The spatial incomplete mode shapes from the dynamical test of grid structure due to quantitative limitation of sensors are then expanded to the complete mode shapes using the mode shape expansion method, which are needed in the damage detection of grid structure based on MSE method.

(2) The damage localization indicator is presented based on MSE method, and both single and multiple damage cases for numerical models of the grid structures are established in different levels of severity, to verify the damage localization detection effectively. However, it fails to localize these members, which are adjacent to each other and are situated in symmetrical areas of the grid structure. The further local information is needed in the future research.

(3) The damage severity estimation algorithm is developed based on the MSE method. In this method, the damage severity column matrix vector $\beta$ in the modified MSE method can be used to estimate the damage severity accurately. The error tolerance value of the column vector is undercontrolled by using the Frobenius norm.
(4) Numerical and physical models of a three-dimensional grid structure are built and have identical geometrical properties. The modified modal strain energy methods for damage localization detection and damage severity detection of the grid structure are implemented with numerical stimulation and lab physical model. Results indicate that the MSE method is effective in damage localization and severity detection of three-dimensional grid structure and can be applied in engineering practice.

\section{Conflicts of Interest}

The authors declare that they have no conflicts of interest.

\section{Acknowledgments}

The authors would like to express their sincere appreciation for the partial financial support from the National Natural Science Foundation of China (Grants nos. 51478459 and 51508554), the Jiangsu Provincial Science and Technology Department (Grant no. BK2011221), and the Social Development Project of Xuzhou Science and Technology Bureau (Grant no. KC16SG270).

\section{References}

[1] Z.-C. Wang and W.-X. Ren, "Crack detection using integrated signals from dynamic responses of girder bridges," Journal of Central South University, vol. 20, no. 6, pp. 1759-1766, 2013.

[2] C.-Q. Miao, Y. Deng, Y.-L. Ding, and A.-Q. Li, "Damage alarming for bridge expansion joints using novelty detection technique based on long-term monitoring data," Journal of Central South University, vol. 20, no. 1, pp. 226-235, 2013.

[3] J.-M. Wang and L.-Z. Chen, "Damage detection of frames using the increment of lateral displacement change," Journal of Zhejiang University: Science, vol. 6, no. 3, pp. 202-212, 2005.

[4] X.-Z. Chen, H.-P. Zhu, and C.-Y. Chen, "Structural damage identification using test static data based on grey system theory," Journal of Zhejiang University: Science, vol. 6, no. 8, pp. 790-796, 2005. 
[5] S. W. Doebling, C. R. Farrar, and M. B. Prime, "A summary review of vibration-based damage identification methods," Shock and Vibration Digest, vol. 30, no. 2, pp. 91-105, 1998.

[6] S. Wang, "Damage detection in offshore platform structures from limited modal data," Applied Ocean Research, vol. 41, pp. 48-56, 2013.

[7] A. Alvandi and C. Cremona, "Assessment of vibration-based damage identification techniques," Journal of Sound and Vibration, vol. 292, no. 1-2, pp. 179-202, 2006.

[8] B. Jaishi and W.-X. Ren, "Finite element model updating based on eigenvalue and strain energy residuals using multiobjective optimisation technique," Mechanical Systems and Signal Processing, vol. 21, no. 5, pp. 2295-2317, 2007.

[9] W.-X. Ren and H.-B. Chen, "Finite element model updating in structural dynamics by using the response surface method," Engineering Structures, vol. 32, no. 8, pp. 2455-2465, 2010.

[10] N. Stubbs, J. T. Kim, and K. Topole, "An efficient and robust algorithm for damage localization in offshore platforms," in Proceedings of the ASCE 10th Structures Congress, pp. 543-556, San Antonio, Tex, USA, 1992.

[11] M. Kumar, R. A. Shenoi, and S. J. Cox, "Experimental validation of modal strain energies based damage identification method for a composite sandwich beam," Composites Science and Technology, vol. 69, no. 10, pp. 1635-1643, 2009.

[12] W. L. Bayissa and N. Haritos, "Structural damage identification in plates using spectral strain energy analysis," Journal of Sound and Vibration, vol. 307, no. 1-2, pp. 226-249, 2007.

[13] H. Li, H. Yang, and S.-L. J. Hu, "Modal strain energy decomposition method for damage localization in 3D frame structures," Journal of Engineering Mechanics, vol. 132, no. 9, pp. 941-951, 2006.

[14] S.-L. J. Hu, S. Wang, and H. Li, "Cross-modal strain energy method for estimating damage severity," Journal of Engineering Mechanics, vol. 132, no. 4, pp. 429-437, 2006.

[15] H. Hu and J. Wang, "Damage detection of a woven fabric composite laminate using a modal strain energy method," Engineering Structures, vol. 31, no. 5, pp. 1042-1055, 2009.

[16] S. Wang, "Iterative modal strain energy method for damage severity estimation using frequency measurements," Structural Control and Health Monitoring, vol. 20, no. 2, pp. 110-120, 2013.

[17] S. Wang, F. Liu, and M. Zhang, "Modal strain energy based structural damage localization for offshore platform using simulated and measured data," Journal of Ocean University of China, vol. 13, no. 3, pp. 397-406, 2014. 


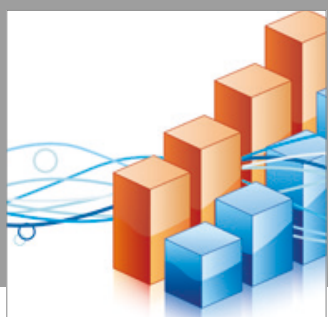

Advances in

Operations Research

vatersals

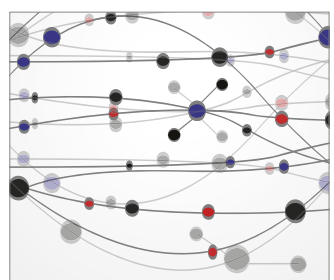

\section{The Scientific} World Journal
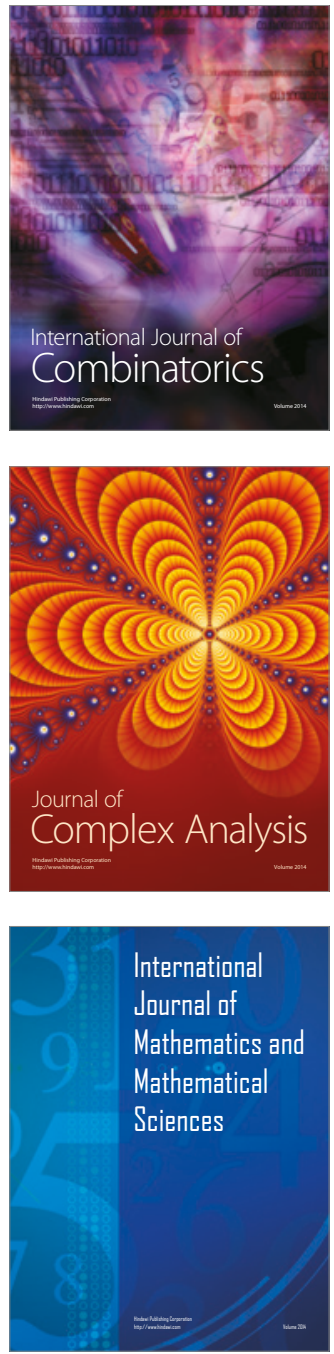
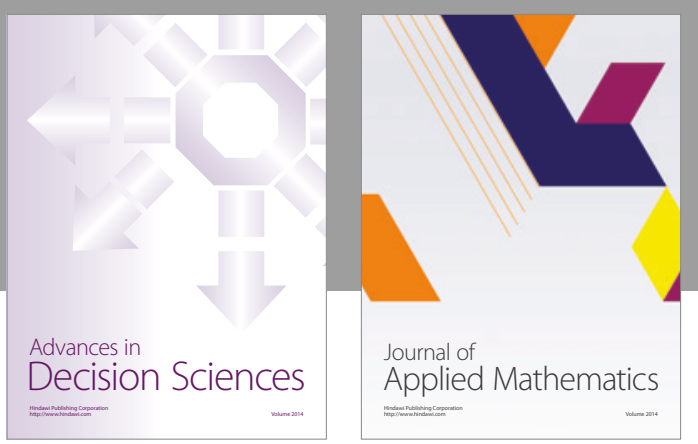

Algebra

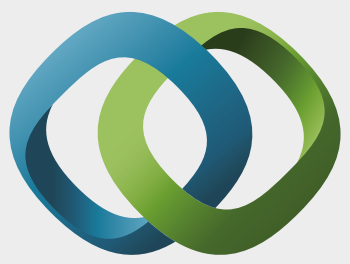

\section{Hindawi}

Submit your manuscripts at

https://www.hindawi.com
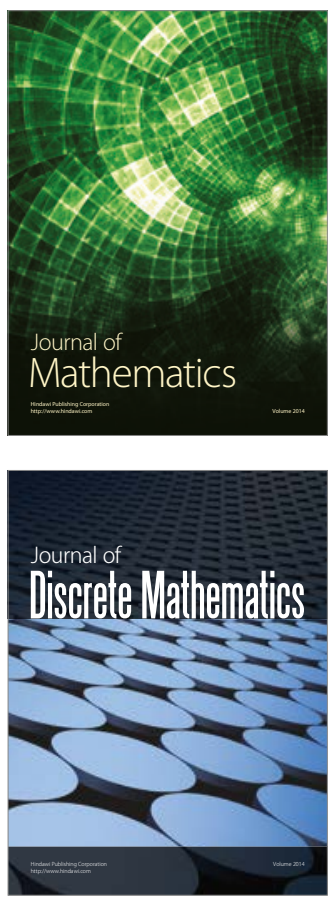

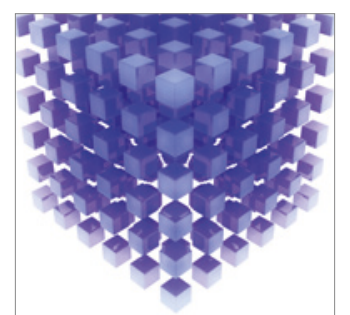

Mathematical Problems in Engineering
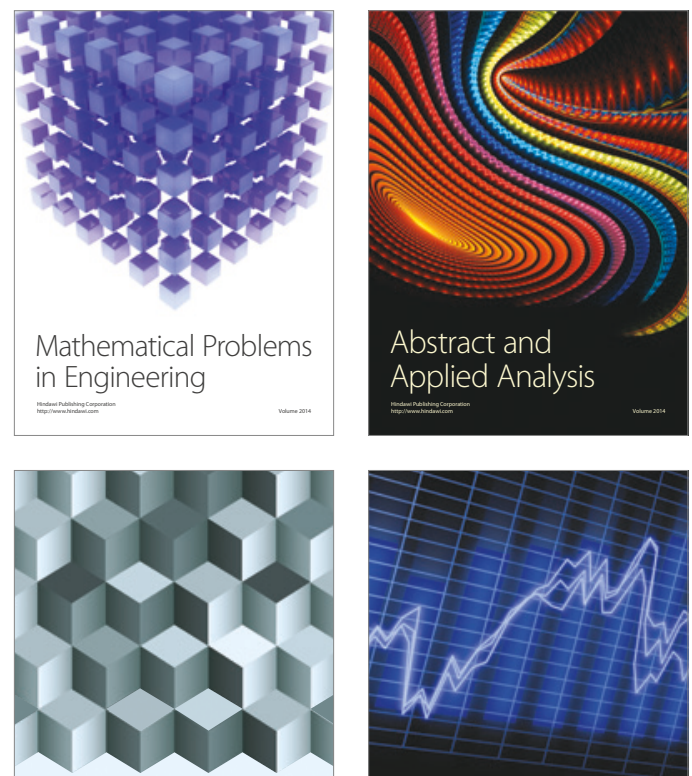

Journal of

Function Spaces

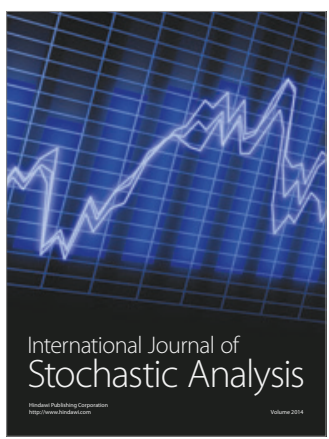

Probability and Statistics
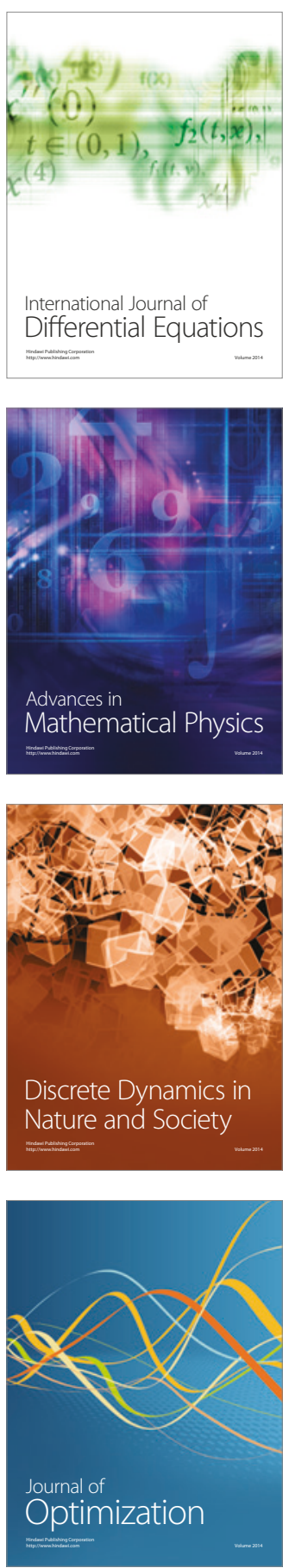